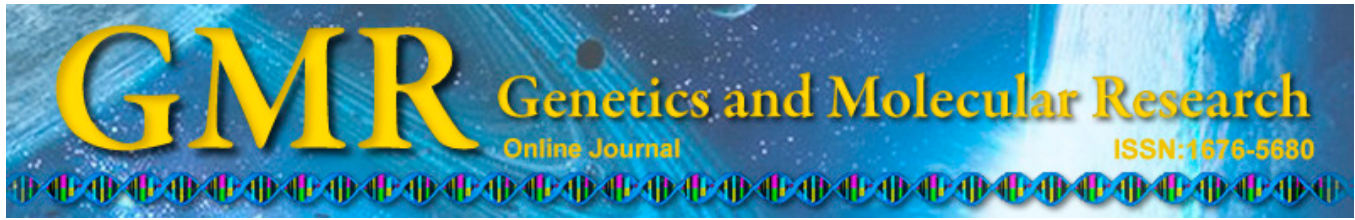

\title{
Effect of chronic hypoxia on penile erectile function in rats
}

\author{
D.P. Yu ${ }^{1}$, X.H. $\mathrm{Liu}^{2}$ and A.Y. Wei ${ }^{3}$ \\ ${ }^{1}$ Department of Urology, \\ The First People's Hospital of Jining City in Shandong Province, \\ Jining, Shandong, China \\ ${ }^{2}$ Shandong Academy of Medical Sciences, Third Affiliated Hospital, \\ Jining, Shandong, China \\ ${ }^{3}$ The First Clinical Medical College, Southern Medical University, \\ Baiyun, Guangzhou, China
}

Corresponding author: D.P. Yu

E-mail: dapengyu_cn@yeah.net

Genet. Mol. Res. 14 (3): 10482-10489 (2015)

Received December 1, 2014

Accepted May 11, 2015

Published September 8, 2015

DOI http://dx.doi.org/10.4238/2015.September.8.9

\begin{abstract}
We examined the relationship between chronic hypoxia and erectile dysfunction in rat and its possible pathogenic mechanism. Forty-eight white male adult Sprague-Dawley rats were randomly divided into a test group and a control group. In accordance with the experimental time $(2,6$, and 10 weeks), each group was divided into 3 subgroups, with 8 rats in each subgroup. Rats in the test group were fed in an airtight hypoxia cabin, while rats in the control group were maintained in a normal environment, with other conditions kept the same. At 2, 6, and 10 weeks, the rats in each group were observed for erectile function. Affinity purification was used to detect neural nitric oxide synthase (nNOS)-positive nerve fibers and endothelial nitric oxide synthase (eNOS) expression. After hypoxia, erectile frequency decreased significantly compared to before hypoxia $(\mathrm{P}<0.001)$. Comparison of the test group and control group revealed a significant difference in the quantity of nNOS-positive nerve fiber and eNOS protein expression $(\mathrm{P}<0.01)$. Hypoxia may influence erectile function
\end{abstract}


and nNOS and eNOS expression in rats. The decrease in the quantity of nNOS nerve fibers and expression of eNOS may contribute to erectile dysfunction under hypoxic conditions in rats.

Key words: Erectile dysfunction; Hypoxia; Nitric oxide synthase; Rat

\section{INTRODUCTION}

Hypoxia, also known as anoxia, refers to the ratio disorders of supply and demand of tissue oxygen. When oxygen supply is reduced, there may not be sufficient oxygen to sustain metabolism or cell structure and function (Siesjoe, 1978; Brierley and Graham, 1985). Lack of oxygen and oxygen utilization disorder may cause hypoxia. Hypoxemia in humans and other animals is visible in hypobaric hypoxia, such as plateau hypoxia, and also visible in normobaric hypoxia caused by respiratory system diseases, such as sleep apnea syndrome. In recent years, approximately $5 \%$ of erectile dysfunction (ED) patients in the andrology clinic of our hospital had sleep apnea syndrome or a recent history of altitude residence, and thus long-term chronic hypoxia may cause sexual dysfunction (Verratti et al., 2011; Budweiser et al., 2013).

To observe penile erectile function in rats, the quantity of neutral nitric oxide synthase (nNOS)-positive fibers in cavernous tissue were measured to explore the mechanism of ED caused by hypoxia.

\section{MATERIAL AND METHODS}

\section{Test materials}

\section{Experimental animals and grouping}

The experimental animals included 48 white male Sprague-Dawley rats provided by the Experimental Animal Center of South Hospital, aged 2 months with body weights of 180$250 \mathrm{~g}$. Rats were randomly divided into 6 groups; based on different experimental times, rats were divided into 2-, 6-, and 10-week control groups and 2-, 6-, and 10-week test groups, with 8 rats in each group. The rats were found to have normal sexual function through mating experiments. The main reagents including monoclonal antibody, anti-eNOS, anti-nNOS, and 3,3'-diaminobenzidine kits were purchased from Beijing Dingguo Biotechnology Limited Liability Company (Beijing, China) and apomorphine hydrochloride (APO) was from Sigma (St. Louis, MO, USA).

\section{Methods}

\section{Preparation of hypoxia model}

The rats were fed in a colorless glass hypoxia cabin with free access to food and the alternation of day and night. Volume of the cabin was $600 \mathrm{~L}$, containing 6 cages and each cage feeding 4 rats. In the module, the built-in temperature hygrometer and 2 window-type air conditioners controlled the cabin at temperature at $22^{\circ}-28^{\circ} \mathrm{C}$ and relative humidity of 50 $60 \%$. The cabin was filled with mixed gas of oxygen and nitrogen to create a $10 \%$ oxygen concentration in the hypoxic environment. Color-changing silica was placed in the hypoxia 
cabin to absorb moisture, and carbon dioxide gas generated by rats respiratory was absorbed by soda lime to maintain the volume of carbon dioxide below $3 \%$ in the cabin. The cage was opened every 3 days, cleaned, and supplied with food and water. The control groups were fed in a normal environment, with other conditions the same as in the experimental group. Rats in each group were sacrificed on the 2nd, 6th, and 10th weekends and the middle penile corpora cavernosa tissues were removed for experiments.

\section{Penile erection experiment}

Rats in each test group were weighed before and after hypoxia and placed in the observation box for 10 min to acclimate. Each rat was injected with APO into the loose skin of the neck. The medication was prepared with saline and $0.5 \%$ vitamin $C$ and administrated at 100 $\mathrm{UG} / \mathrm{kg}$. After medication, the rats were immediately observed for $30 \mathrm{~min}$ to record whether the penis was erectile and to determine erectile frequency. Penile glans hyperemia and the emerging end of the penis were taken as the time of erection.

\section{Detection of nNOS staining positive nerve fibers}

On the 2nd, 6th, and 10th weekends, rats in each groups were taken anatomically isolated penile tissue, removed skin, glans penis, cavernous body of urethra and penis bone, and the middle of the cavernosal tissue was divided into 3 equal parts for nNOS, eNOS, and pathological examination. Routine paraffin embedding, slicing, and immunohistochemical SP method was used to detect the number of nNOS-positive nerve fibers.

\section{Expression of eNOS}

Phosphate-buffered saline solution rather than primary antibody was used as a negative control, while a known positive section was used as a positive control. The cytoplasm and the nucleus containing brown-yellow granules represented positive expression. eNOS was quantitatively analyzed using an HPZAS-1000 high-definition color pathological image analysis system to determine absorbance values, and the mean values were compared.

\section{Data statistics}

Results are reported as means \pm standard deviation (means \pm SD) and compared using the paired-sample $t$-test. Multiple comparisons between groups used analysis of variance, and statistical analysis used the SPSS 10 software (SPSS, Inc., Chicago, IL, USA).

\section{RESULTS}

\section{Establishment of model and erection of penis experiment}

After only 1 day in the hypoxia cabin, rats in the test groups showed obvious hypoxia symptoms, such as shortness of breath, anorexia, lethargy, slow weight growth, and weight loss.

All test groups of rats were injected with APO at the 2nd, 6th, and 10th weekends and were evaluated for erectile function (Table 1). Compared with each experimental group before and after hypoxia, penile erection times decreased significantly $(\mathrm{P}<0.001)$, indicating that the 
hypoxia experimental animal model had been successfully established.

Erectile frequency for each test group rats before and after hypoxia in the 2-week group, t-test $=7.937, \mathrm{df}=7, \mathrm{P}=0.001$ (bilateral), showing a statistically significant difference, indicating that compared with before hypoxia, the penile erectile frequency in rats decreased after hypoxia for 2 weeks (before and after hypoxia, the mean $\mathrm{D}$ value of erectile times difference was 1.5). In the 6-week group, t-test $=9.105, \mathrm{df}=7, \mathrm{P}=0.001$ (bilateral), and the difference was statistically significant, indicating that compared with before hypoxia, the penile erectile frequency in rats decreased after hypoxia for 2 weeks (before and after hypoxia, the mean D-value of erectile frequency was 3.75). In the 10 -week group, t-test $=7.483, \mathrm{df}=7, \mathrm{P}=$ 0.001 (bilateral), and the difference was statistically significant, indicating that compared with before hypoxia, the penile erectile times in rats decreased after hypoxia for 2 weeks (before and after hypoxia, the mean $\mathrm{D}$ value of erectile times was 2 ).

Table 1. Erectile frequency in each test subgroup (means \pm SD).
\begin{tabular}{lccc}
\hline Experience group & Cases number & Erectile times before hypoxia & Erectile times after hypoxia \\
\hline 2-week group & 8 & $5.4 \pm 2.1$ & $3.9 \pm 1.8$ \\
6-week group & 8 & $5.5 \pm 1.8$ & $1.6 \pm 1.0$ \\
10-week group & 8 & $5.5 \pm 2.2$ & $3.5 \pm 1.9$ \\
\hline
\end{tabular}

Comparison of the erectile frequency in each test group rats before and after hypoxia showed that in addition to at the 2nd and 10th weekends, the difference between the 2 groups was not significant $(\mathrm{P}=0.257)$. The difference between other groups was significant $(\mathrm{P}<0.01)$, indicating that influence of hypoxia for 6 weeks on erectile function was more severe than hypoxia for 2 and 10 weeks, respectively. Comparison of hypoxia for 10-week group with the hypoxia for 2-week group showed that the difference was not significant $(\mathrm{P}=0.257)$, indicating that erectile function in rats may be partially compensated after hypoxia for 10 weeks.

Comparison of the erectile frequency in each test group before and after hypoxia showed that the homogeneity of variance was significant, indicating that different hypoxia times had varying effects on the degree of erectile frequency.

\section{LSD test results}

In addition to the 2- and 10-week groups, compared to other groups, the D value of the erectile frequency of rats before and after hypoxia was statistically different. The 6 weeks group showed the highest values, indicating that hypoxia for 6 weeks had a greater influence on erectile function in rats than in the hypoxia for 2-week group $(\mathrm{P}=0.000)$ and hypoxia for 10week group $(\mathrm{P}=0.001)$, while there was no significant difference $(\mathrm{P}=0.257)$ of the influence on erectile function between the hypoxia for 2-week group and hypoxia for 10-week group.

\section{Count of nNOS-positive nerve fibers}

The quantity of nNOS staining-positive nerve fibers in rat penile tissue in the test group was significantly lower than that in the control group $(\mathrm{P}<0.01)$. In a comparison of the 2-week hypoxia group with the 2-week control group, $\mathrm{t}$-test $=2.667, \mathrm{P}=0.018$, comparison the 6-week hypoxia group and the 6-week control group, t-test $=8.176, \mathrm{P}=0.000$ (Figure 1A), and comparison of the 2 -week hypoxia group with the 2 -week control group, $\mathrm{t}$-test $=10.024, \mathrm{P}$ 
$=0.000$ (Figure 1B) showed that the quantity of nNOS staining-positive nerve fibers were not significantly different between the control groups.

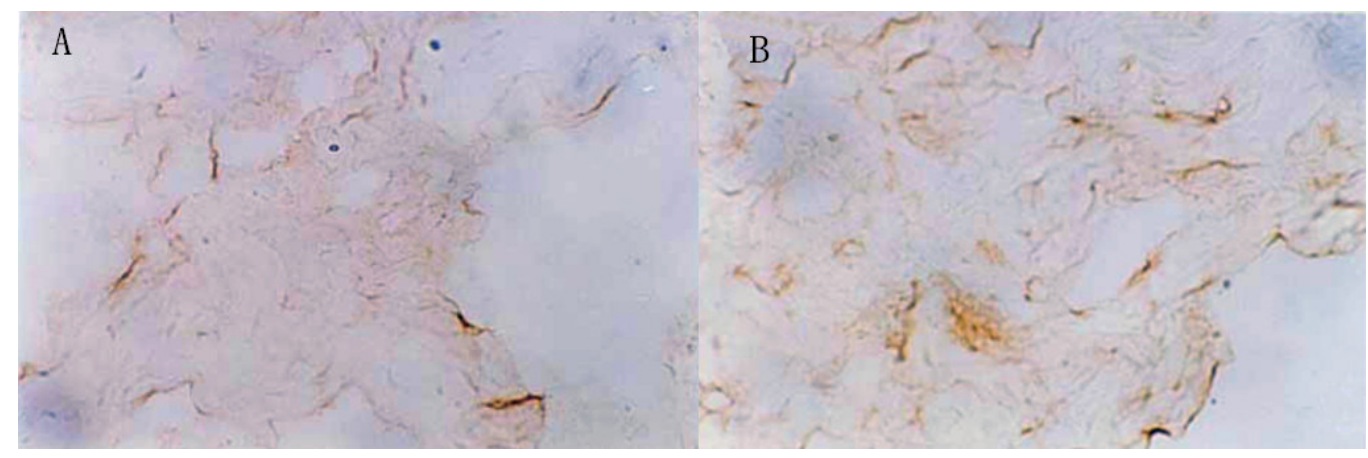

Figure 1. Expression of nNOS-positive nerve fibers in corpus cavernosum in 6- and 10-week group of the test group. A. Expression of nNOS-positive nerve fibers in corpus cavernosum in 6-week group of the test group. B. Expression of nNOS-positive nerve fibers in corpus cavernosum in 10-week group of the test group.

The quantity of nNOS staining-positive nerve fibers in the 6-week test group was significantly lower than that in the 2 -week test group ( $\mathrm{t}$-test $=6.624, \mathrm{P}=0.000)$. Comparison of the 6-week test group with the 10-week test group showed that the quantity of nNOS stainingpositive nerve fibers was significantly different ( $\mathrm{t}$-test $=-3.003, \mathrm{P}=0.014<0.05)$. Comparison of the 2-week test group with the 10 -week test group showed that the quantity of nNOS staining-positive nerve fibers was significantly different ( $\mathrm{t}$-test $=6.444, \mathrm{P}=0.000)$. With prolonged hypoxia time, the quantity of nNOS staining-positive nerve fibers gradually increased (Table 2).

Table 2. Quantity of nNOS-positive nerve fibers in corpus cavernosum in control and test group (means \pm SD).

\begin{tabular}{lcc}
\hline Group & Case number & No. of nNOS staining positive nerve fibers \\
\hline Contrast 2-week group & 8 & $106.88 \pm 20.40$ \\
Contrast 6-week group & 8 & $96.38 \pm 7.745$ \\
Contrast 10-week group & 8 & $105.75 \pm 9.27$ \\
Experiment 2-week group & 8 & $86.75 \pm 6.27$ \\
Experiment 6-week group & 8 & $54.75 \pm 12.13$ \\
Experiment 10-week group & 8 & $68.75 \pm 4.80$ \\
\hline
\end{tabular}

\section{Expression of eNOS protein}

eNOS is mainly expressed in the corpus cavernosum arteries, in the nucleus, and cytoplasm of the blood sinus endothelial cells, and the positive expression showed a yellow brown granular scattering distribution. eNOS-positive expression values detected by the image analysis measurement system are shown in Table 3. Comparison of the 2-week hypoxia group with the 2-week control group, t-test $=4.039, \mathrm{P}=0.001$, and comparison of the 6 weeks hypoxia group with the 6-week control group, t-test $=10.748, \mathrm{P}=0.000$ (Figure 2A) showed 
that eNOS-positive expression in the test group was significantly lower than that in the control group. Comparison of the 10-week hypoxia group and the 10-week control group, t-test $=$ $1.413, \mathrm{P}=0.179$, showed no statistical significance, indicating no difference in eNOS expression between the hypoxia for 10-week control groups (Figure 2B).

Table 3. Expression of eNOS protein in corpus cavernosum in control and test group (means $\pm \mathrm{SD}$ ).

\begin{tabular}{lcc}
\hline Group & Case number & Expression of eNOS protein \\
\hline Contrast 2-week group & 8 & $0.1017 \pm 0.0097$ \\
Contrast 6-week group & 8 & $0.0961 \pm 0.0062$ \\
Contrast 10-week group & 8 & $0.0935 \pm 0.0345$ \\
Experiment 2-week group & 8 & $0.0860 \pm 0.0052$ \\
Experiment 6-week group & 8 & $0.0668 \pm 0.0046$ \\
Experiment 10-week group & 8 & $0.0761 \pm 0.0045$ \\
\hline
\end{tabular}

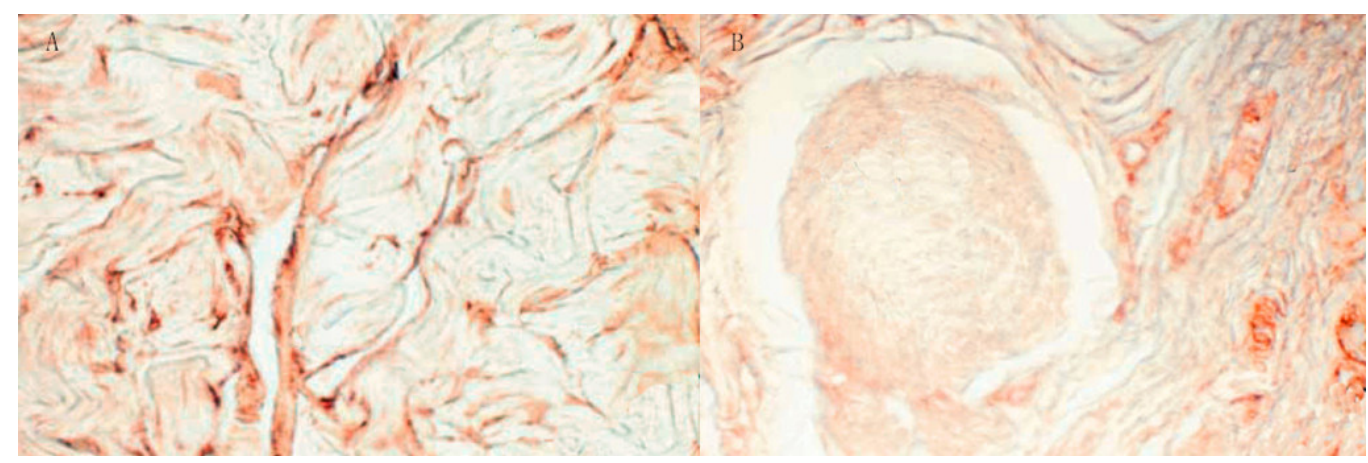

Figure 2. Expression of eNOS protein in corpus cavernosum in 6- and 10-week group of the test group. A. Expression of eNOS protein in corpus cavernosum in 6-week group of the test group. B. Expression of eNOS protein in corpus cavernosum in 10 -week group of the test group.

In the test group, eNOS expression was high in the 2-week group, decreased in the 6-week group, and then increased in the 10-week groups. The differences were significant ( $\mathrm{F}$ $=32.260, \mathrm{P}<0.01$ ), indicating that with the duration of hypoxia, eNOS protein expression decreased first and increased again.

\section{DISCUSSION}

ED had a higher incidence in male patients with chronic hypoxemia (Fanfulla et al., 1989). Hirshkowitz et al. (1989) found that in patients with sleep apnea syndrome complicated with ED, the apnea index is negatively related to penile rigidity (Seftel et al., 2002). However, its pathogenesis has remained unclear. Because the cases are from clinical patients, specimens are difficult to obtain, the clinical research may be influenced by subjectivity, and the lack of controls, establishing a model of hypoxic ED was necessary for in-depth study.

During the erection process, relaxation of penile vascular and cavernosal smooth muscle is an important step in erection, and this relaxation is completed under regulation of the nonadrenergic noncholinergic nerve and cholinergic nerve-sinusoidal endothelial system, 
through the L-arginine nitric oxide cGMP pathway. In this pathway, nitric oxide released by nonadrenergic noncholinergic neural plays a key role in penile erection, which is produced by the conversion of L-arginine catalyzed by NOS. There are 2 types of NOS penile tissue, including nNOS and eNOS, in which the former mainly catalyzes NO production from Larginine (Magge et al., 1996).

Using the experimental ED animal model established in a hypoxic environment, an immunohistochemical method was used to detect the quantity of nNOS staining-positive nerve fiber and expression of eNOS protein in penile tissue. With the duration of hypoxia, the total expression of nNOS and eNOS decreased, indicating that the pathogenesis of ED may be related to the reduced generation of NO caused by decreased NOS. In addition, erectile function in the hypoxia for 6-week group was better than in the hypoxia for 10-week group. Expression of nNOS and eNOS in the 10-week group was higher than that in the 6-week group, and eNOS in hypoxia 10-week group and control group showed no significant difference. This indicates that with prolonged hypoxia time, erectile function may be partially compensated, explaining why the erectile function in the 10-week group was better than that in the 6-week group.

In the hypoxic environment, ED may be the result of many factors, including a series of neural, humoral, and cellular factors.

Because the normal biological activity of NOS depends on oxygen molecules, the biosynthesis of NO is restrained under anaerobic conditions and the vasoconstrictor (norepinephrine, endothelin) is dominant, limiting the diastolic of corpus cavernosum trabecular and resulting in a persistent flaccid state (Kim et al., 1993; Palmer et al., 1994; Christ, 1995; Nehra et al., 1996; Burnett, 1997; Traish et al., 1997; Moreland, 1998; Moreland et al., 2001, 2002; Prieto et al., 2010; Liu et al., 2012). Synthesis of cytokines, growth factors, internal secretion, and vasoactive factors is affected by oxygen partial pressure, and these factors play an important role in the metabolism of smooth muscle cells and connective tissue (Traish, 1997), making the ratio of functional connective tissue/muscle maintained in the normal range to ensure the normal erectile of the penis (Gao et al., 1996; Traish, 1997). Thus, under chronic hypoxic conditions, cavernous endothelial cell oxygenation obstacles, and endothelial damage, resulting in synthesis of connective tissue, accelerate the formation of cavernous fibrosis and vein closing mechanism disorder.

In summary, the occurrence of hypoxic ED is a complex process involving hemodynamics, and low oxygen partial pressure as a direct cause through the mutual action of multiple factors of nerves, endocrine hormones, humoral factors, and cell metabolism, resulting in reduced NOS expression and cell ultrastructure pathological changes, which have a negative impact on erectile function. These factors ultimately lead to the occurrence of ED. Because of the limitations of our experimental conditions, we could not conduct in-depth analysis of NOS. Under long-term chronic hypoxic conditions, decreased expression of NOS may be the mechanism of ED. Over a prolonged time of hypoxia, erectile function may be partially compensated. Additionally, whether other neurohumoral, cytokine, and hypoxic conditions have pathological effects on ED requires further analysis.

\section{Conflicts of interest}

The authors declare no conflict of interest. 


\section{REFERENCES}

Brierley JB and Brown AW (1982). The origin of lipid phagocytes in the central nervous system: II. The adventitia of blood vessels. J. Comp. Neurol. 211: 407-417.

Brierley JB and Graham DL (1985). Hypoxia and vascular disorders of the central nervous system. In: Greenfield's Neuropathology (Blackwood W and Corsellis AN, eds.). Martin Dunita, London, 125-156.

Budweiser S, Luigart R, Jörres RA, Kollert F, et al. (2013). Long-term changes of sexual function in men with obstructive sleep apnea after initiation of continuous positive airway pressure. J. Sex. Med. 10: 524-531.

Burnett AL (1997). Nitric oxide in the penis: physiology and pathology. J. Urol. 157: 320-324.

Christ GJ (1995). The penis as a vascular organ. The importance of corporal smooth muscle tone in the control of erection. Urol. Clin. North Am. 22: 727-745.

Fanfulla F, Malaguti S, Montagna T, Salvini S, et al. (1989). Erectile dysfunction in men with obstructive sleep apnea: an early sign of nerve involvement. Sleep 23: 775-781.

Gao H, Korthuis RJ and Benoit JN (1996). Hypoxia/reoxygenation selectively impairs alpha 1b-adrenoceptor function in small mesenteric arteries. Am. J. Physiol. 271: G820-G823.

Hirshkowitz M, Karacan I, Gurakar A and Williams RL (1989). Hypertension, erectile dysfunction, and occult sleep apnea. Sleep 12: 223-232.

Kim N, Vardi Y, Padma-Nathan H, Daley J, et al. (1993). Oxygen tension regulates the nitric oxide pathway. Physiological role in penile erection. J. Clin. Invest. 91: 437-442.

Liu K, Liu XS, Xiao L, Shang J, et al. (2012). NADPH oxidase activation: a mechanism of erectile dysfunction in a rat model of sleep apnea. J. Androl. 33: 1186-1198.

Magge M, Funetes AM, Garban H, Rajavashisth T, et al. (1996). Cloning of a novel neuronal nitric oxide synthase expressed in penile and lower urinary tract. Biochem. Biophys. Res. Commun. 226: 145-151.

Moreland RB (1998). Is there a role of hypoxemia in penile fibrosis: a viewpoint presented to the Society for the Study of Impotence. Int. J. Impot. Res. 10: 113-120.

Moreland RB, Albadawi H, Bratton C, Patton G, et al. (2001). O2-dependent prostanoid synthesis activates functional PGE receptors on corpus cavernosum smooth muscle. Am. J. Physiol. Heart Circ. Physiol. 281: H552-H558.

Moreland RB, Nehra A, Kim NN, Min KS, et al. (2002). Expression of functional prostaglandin D (DP) receptors in human corpus cavernosum smooth muscle. Int. J. Impot. Res. 14: 446-452.

Nehra A, Goldstein I, Pabby A, Nugent M, et al. (1996). Mechanisms of venous leakage: a prospective clinicopathological correlation of corporeal function and structure. J. Urol. 156: 1320-1329.

Palmer LS, Valcic M, Melman A, Giradli A, et al. (1994). Characterization of cyclic AMP accumulation in cultured human corpus cavernosum smooth muscle cells. J. Urol. 152: 1308-1314.

Prieto D, Kaminski PM, Bagi Z, Ahmad M, et al. (2010). Hypoxic relaxation of penile arteries: involvement of endothelial nitric oxide and modulation by reactive oxygen species. Am. J. Physiol. Heart Circ. Physiol. 299: H915-H924.

Seftel AD, Strohl KP, Loye TL, Bayard D, et al. (2002). Erectile dysfunction and symptoms of sleep disorders. Sleep 25: 643-647.

Siesjoe BK (1978). Hypoxia in Brain Energy Metabolism. Chapter 14. Toronto, New York, 398-452.

Traish AM, Moreland RB, Gallant C, Huang YH, et al. (1997). G-protein-coupled receptor agonists augment adenylyl cyclase activity induced by forskolin in human corpus cavernosum smooth muscle cells. Recept. Signal Transduct. 7: 121-132.

Verratti V, Falone S, Fanò G, Paoli A, et al. (2011). Effects of hypoxia on nocturnal erection quality: a case report from the Manaslu expedition. J. Sex. Med. 8: 2386-2390. 